\title{
ORIGINAL ARTICLE \\ Sympathetic single axonal discharge after spinal cord injury in humans: activity at rest and after bladder stimulation
}

\author{
BG Wallin ${ }^{1}$, T Karlsson ${ }^{1}$, G Pegenius ${ }^{1}$, A-K Karlsson ${ }^{2}$, VG Macefield ${ }^{3}$ and M Elam ${ }^{1}$
}

\begin{abstract}
Study design: Clinical experimental mechanistic study.
Objectives: (1) To determine in three spinal cord-injured patients whether individual muscle sympathetic nerve fibres below the level of the spinal lesion display spontaneous activity. (2) To determine in these patients if individual sympathetic vasoconstrictor fibres show a prolonged discharge following a bladder stimulus.

Setting: University hospital in Gothenburg, Sweden.

Methods: Microneurographic recordings of action potentials from individual muscle nerve sympathetic fibres in a peroneal nerve. Recordings of skin blood flow and electrodermal responses in a foot.

Results: In all patients, there was sparse ongoing spontaneous impulse traffic in individual sympathetic fibres. Brisk mechanical pressure over the urinary bladder evoked a varying number of action potentials in individual fibres, but the activity was brief and did not continue after the end of the evoked multiunit burst.
\end{abstract}

Conclusion: Prolonged discharges in individual sympathetic fibres are unlikely to contribute to a long duration of blood pressure increases induced by brief bladder stimuli.

Spinal Cord (2014) 52, 434-438; doi:10.1038/sc.2014.35; published online 25 March 2014

Keywords: sympathetic nerve activity; spinal cord injury; bladder stimulation

\section{INTRODUCTION}

High-level spinal cord injury (SCI) that interrupts descending sympathetic pathways leads to low resting arterial pressure and postural hypotension. Still, sensory stimuli originating from the viscera, such as the bladder or the colon, or somatosensory inputs below the lesion, can trigger marked vasoconstriction and dangerous increases in arterial pressure-autonomic dysreflexia. Microelectrode recordings of multifibre sympathetic nerve activity below the level of the lesion in patients with such lesions revealed negligible resting sympathetic outflow to blood vessels in skin $^{1}$ and muscle. ${ }^{2}$ In agreement with this, resting blood flow was also high below the lesion in SCI patients. Application of pressure over the bladder, skin pinches or electrical skin stimuli below the lesion evoked only modest increases of multifibre sympathetic activity in the integrated neurogram, ${ }^{1,2}$ but, nevertheless, large increases of noradrenaline spillover below the lesion and marked increases in blood pressure ${ }^{3}$ have been reported.

Two main alternatives have been put forward to explain the discrepancy between the weak stimuli-induced responses in sympathetic activity on one hand, and the exaggerated effects on noradrenaline spillover and blood pressure (BP) on the other. One possibility is that in SCI patients the neural response to a bladder stimulus is not only the brief initial burst of impulses seen in the integrated neurogram, but also a continuing discharge of desynchronized activity following the burst. If so, the consequence would be that after the initial burst the integrated neurogram appears flat, that is, falsely with low activity. To decide if this is the case requires information on impulse traffic in individual sympathetic fibres.

The other alternative is that the modest multiunit bursts seen after a bladder stimulus cause an exaggerated release of noradrenaline from the sympathetic terminals and/or that the blood vessels have become hypersensitive to the transmitter(s). There is supporting evidence for the second alternative, ${ }^{3-5}$ but recordings from single sympathetic fibres have not been reported in humans with SCI.

Against this background, the aim of the present study was to record impulse activity from individual sympathetic fibres to the muscle below the level of the lesion in SCI patients, both at rest and in response to bladder stimulation. We asked the following questions: (1) is there spontaneous sympathetic impulse traffic at rest? (2) Does the brief multiunit burst of neural activity evoked by bladder stimuli contain single-unit 'salvos' with high instantaneous firing frequencies? (3) Do bladder stimuli evoke continuing desynchronized single-fibre activity after the initial burst of impulses?

\section{MATERIALS AND METHODS}

\section{Patients}

Recordings were made from two men and one woman aged 29-45 (mean 38) with SCI at levels C5-C7, American Spinal Injury Association Impairment Scale (AIS) grade A. None of the patients suffered from decubitus ulcers and they had a stable regular bowel regimen without impaction, using laxatives occasionally. Two patients received oral calcium carbonate and risedronic acid to prevent osteoporosis. The third patient received oral methenamine to

${ }^{1}$ Department of Clinical Neurophysiology, Institute of Neuroscience and Physiology, Sahlgren Academy at University of Gothenburg, Sahlgren University Hospital, Gothenburg, Sweden; ${ }^{2}$ Department of Neurology, Institute of Neuroscience and Physiology, Sahlgren Academy at University of Gothenburg, Gothenburg, Sweden and ${ }^{3}$ School of Medicine, University of Western Sydney, Sydney, NSW, Australia

Correspondence: Dr BG Wallin, Department of Clinical Neurophysiology, Institute of Neuroscience and Physiology, Sahlgren Academy at University of Gothenburg, Sahlgren University Hospital, S-413 45 Gothenburg, Sweden.

E-mail: gunnar.wallin@neuro.gu.se

Received 11 December 2013; revised 24 February 2014; accepted 28 February 2014; published online 25 March 2014 
prevent bladder infections, and intermittent intrathecal infusions of baclofen against spasticity.

In accordance with the Declaration of Helsinki, each subject provided informed written consent to the procedures, which were approved by the human ethics committee of the University of Gothenburg, Sweden, where the experiments were performed.

\section{Measurements}

Electrocardiogram was recorded by $\mathrm{Ag}-\mathrm{AgCl}$ electrodes on the chest and respiratory movements by a strain-gauge belt around the upper part of the abdomen. Arterial finger blood pressure was monitored continuously by the volume-clamp method (Finometer, Arnhem, The Netherlands), with the cuff around the middle phalanx of the third finger on the same side as the microneurography recording. Electrodermal changes were recorded by $\mathrm{Ag}-$ $\mathrm{AgCl}$ surface electrodes placed on glabrous skin in a hand and a foot. The measuring current was $12 \mu \mathrm{A}$ and the bandwidth $0.7-100 \mathrm{~Hz}$. Changes in skin blood flow were monitored by laser-doppler flow probes (Periflux 4001, Perimed AB, AQSweden) applied to glabrous skin on one hand and foot. The instrument was calibrated with respect to the minimum and maximum electrical signals at a constant gain setting and expressed as 0-1000 perfusion units.

Muscle sympathetic nerve activity was recorded with a high-impedance microelectrode (type 25-10-1, Frederick Haer Co., Brunswick, ME, USA; or type TM33B20, World Precision Instruments, Sarasota, FL, USA) inserted into a motor fascicle of the peroneal nerve. A low-impedance reference electrode was inserted subcutaneously, a few centimeters away from the recording electrode. During the experiment, neural activity and effector signals were monitored on a storage oscilloscope and also displayed on two computer screens, one guiding the microneurographer and the other guiding data collection.

\section{Procedure}

A muscle nerve fascicle was identified by afferent responses evoked by muscle taps and muscle stretch, but not by skin stroking. When such a site was found, a leg muscle was pinched below the level of the lesion or a brisk shortlasting pressure was applied over the bladder. If no multiunit responses were obtained, a small electrode adjustment was made and the stimuli were reapplied. When stimuli evoked multiunit responses followed by signs of vasoconstriction or skin resistance reduction in the lower leg, a sympathetic site was considered to be present. ${ }^{2}$ If necessary, further needle adjustments were made until a site was obtained in which the stimuli evoked putative single-fibre potentials.

\section{Data collection}

Neural activity was amplified $(\times 50000$ ), filtered (band pass $300-6000 \mathrm{~Hz}$ ), digitized at 10 or $20 \mathrm{kHz}$ (16 bits) and stored on disk via a PowerLab 16SP data acquisition computer system and Chart analysis software (AD Instruments Pty Ltd, Bella Vista, NSW, Australia). The amplified and filtered nerve signal was also fed through a discriminator for further noise reduction and audiomonitoring. A mean-voltage (integrated) neurogram (time constant $0.1 \mathrm{~s}$ ) was digitized at $200 \mathrm{~Hz}$ and stored as 16 bits. The electrocardiogram channel was digitized at $800 \mathrm{~Hz}$ (16 bits), and respiration and arterial pressure at $200 \mathrm{~Hz}$ (16 bits). Data were sampled in standard epochs of $300 \mathrm{~s}$ duration.

\section{Analysis}

The original neurogram signal was exported in Matlab (The MathWorks, Inc., Natick, MA, USA) format, which was used for all subsequent analyses, using laboratory-developed routines. These include a $50-\mathrm{Hz}$ notch filter to reduce power line interference, a matched filter to detect individual spike shapes and display routines for simultaneous presentation of segments of the neural activity at different time resolutions.

Each recording was inspected by a single investigator. All individual action potentials with a common waveform were collected in a separate window, in which the potentials were superimposed at an individually selected time resolution. When action potentials of different waveforms were present, several such windows were used. To be accepted as an action potential from an unmyelinated fibre, the potential should have a main negative deflection. ${ }^{6}$ Each accepted potential was characterized quantitatively in terms of amplitude, duration and slope of the fast phase of the main negative deflection. The amplitude was measured as the negative phase of the potential (peak to minimum) and expressed in relation to the noise level. The duration was calculated as the full width at half maximum of the amplitude. The slope was set to the gradient of the lower half of the downstroke of the negative phase of the spike. To consider such potential deflections as action potentials from a single nerve fibre, our criterion was that the potential should occur at least four times with a reproducible waveform during a recording in an unchanged electrode site. Furthermore, the amplitude of the action potential had to exceed six times the noise level, which was defined as the s.d. of a few seconds duration of the nerve signal that appeared to contain no action potentials. The duration of a multiunit response to bladder stimulation was determined from the mean-voltage neurogram.

We certify that all applicable institutional and governmental regulations concerning the ethical use of human volunteers were followed during the course of this research.

\section{RESULTS}

\section{Multiunit activity}

The mean-voltage neurogram of the three SCI subjects did not display spontaneously occurring multiunit bursts during baseline rest. In agreement with previous reports from SCI patients, ${ }^{1,2}$ brisk manual pressure applied over the urinary bladder often evoked a single multiunit burst of impulses followed by signs of cutaneous vasoconstriction and/or decreases in skin resistance in the hands and/or in the feet. In addition, multiunit neural bursts and cutaneous effector responses occurred in association with spasticity in the muscles of the legs, which was occasionally evoked during the recording procedure.

\section{Single-fibre potentials}

In all the three patients, the original neurogram contained a varying degree of irregularly occurring potentials from 29, 21 and 12 (total 62) individual nerve fibres, respectively (Table 1).

The waveforms of the potentials were similar to those of the sympathetic action potentials described previously in able-bodied healthy subjects, ${ }^{6,7}$ but the durations varied between potentials from different fibres (Figure 1).

At rest, the potentials had mean frequencies of $0.08,0.09$ and $0.03 \mathrm{~Hz}$, respectively, in the three patients; no clear cardiac rhythmicity was detected.

\section{Single-fibre responses to bladder stimuli}

Bladder stimuli were applied, respectively, 15, 15 and 10 (total 40) times in the three patients, and acceptable single-fibre responses associated with signs of vasoconstriction in the laser-doppler flow meter were obtained 13, 10 and 2 times, respectively. Examples of unitary responses to bladder stimuli are shown in Figure 1 and details of the responses in the respective SCI patients are given in Table 2.

\section{Table 1 Resting activity}

\begin{tabular}{lccc}
\hline & \multicolumn{3}{c}{ SCl patients } \\
\cline { 2 - 4 } & UC & $H H$ & $P L$ \\
\hline Analysed time (min) & 34 & 18 & 34 \\
Total no. of active fibres at rest & 29 & 21 & 12 \\
Total no. of spikes per min, all fibres & $12.0 \pm 6.0$ & $22.7 \pm 9.1$ & $3.5 \pm 2.0$ \\
Mean frequency per fibre (Hz) & $0.08 \pm 0.06$ & $0.09 \pm 0.03$ & $0.03 \pm 0.02$
\end{tabular}

Abbreviation: $\mathrm{SCl}$, spinal cord injury. 

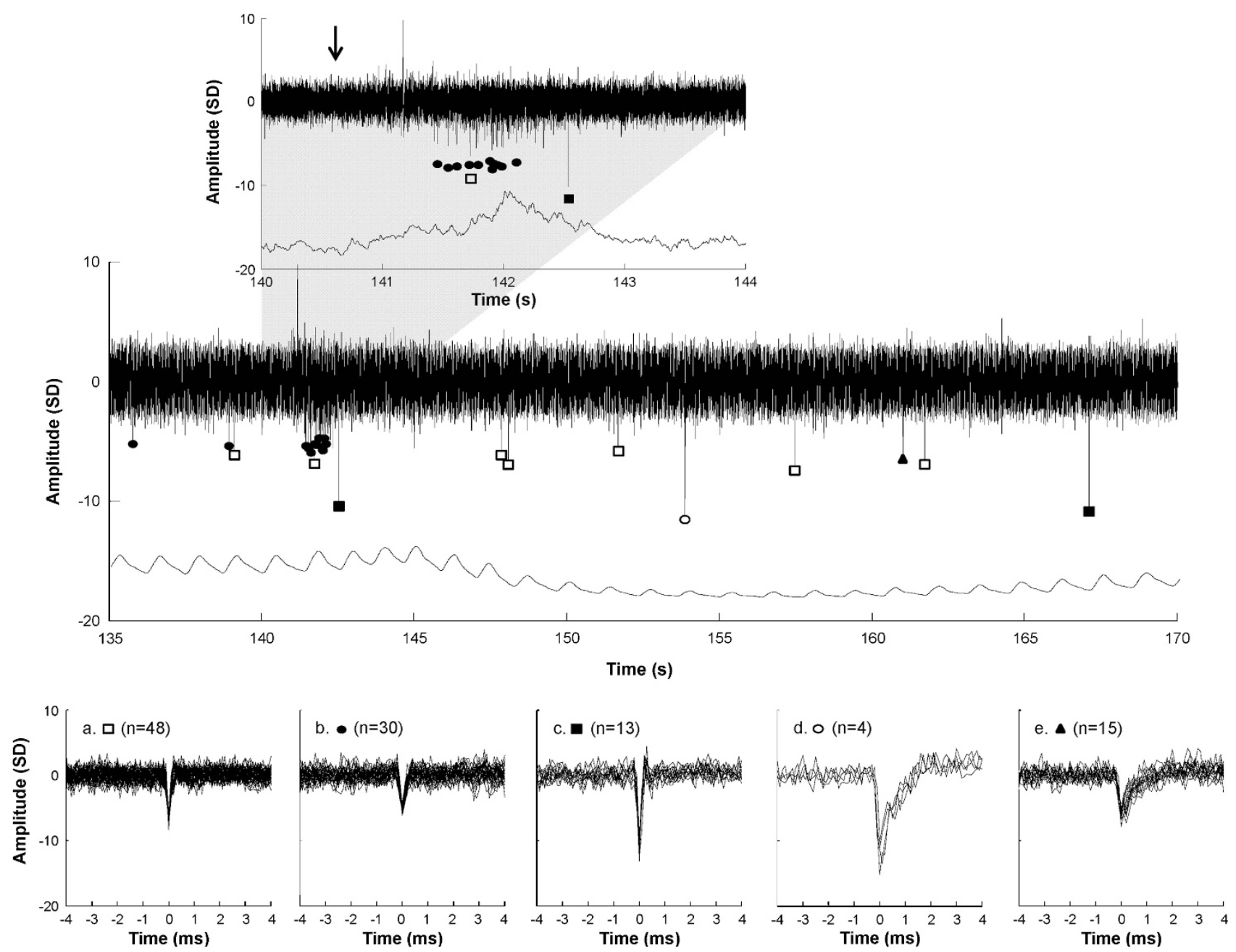

Figure 1 Middle panel: Original neurogram and laser-doppler flow record from SCl patient HH. Grey-shaded inset (above the middle panel) shows responses in original and integrated neurograms to a bladder stimulus (arrow) on an expanded time scale. Lower panels show superimpositions of action potentials with five different waveforms occurring during a recording of $8.3 \mathrm{~min}$. The single-unit response to the stimulus shown in the figure contained potentials of the three left waveforms occurring (from left) 1,12 and 1 times, respectively. The potential in panel a differs significantly $(P<0.001)$ from those in panels $\mathbf{b}$ and $\mathbf{c}$ with regard to amplitude, duration and slope. The potentials in panels $\mathbf{b}$ and $\mathbf{c}$ differ significantly $(P<0.001)$ with regard to amplitude and slope but not with regard to duration $(P>0.05)$. Note that the high-amplitude positive deflection occurring after the stimulus is an artefact.

Table 2 Responses to bladder stimulation

\begin{tabular}{lccc}
\hline & \multicolumn{3}{c}{ SCl patient } \\
\cline { 2 - 4 } & UC & $H H$ & $P L$ \\
\hline No. of bladder stimuli & 13 & 10 & 2 \\
Multiunit response duration (s) & $1.2 \pm 0.3$ & $1.8 \pm 0.3$ & 1.2 \\
No. of responding fibres & $1.6 \pm 0.7$ & $2.3 \pm 1.4$ & 1.5 \\
Maximum no. of spikes in any fibre & $8(97 / 57)$ & $12(99 / 35)$ & $6(45 / 24)$ \\
(max/mean instantaneous frequency (Hz)) & & & \\
Total no. of spikes in response & $4.5 \pm 2.7$ & $6.2 \pm 4.0$ & 5.5 \\
No. of spikes in 10 s before response & $1.7 \pm 1.9$ & $4.3 \pm 2.0$ & 1.5 \\
No of spikes in 10s after response & $1.7 \pm 1.5$ & $3.4 \pm 1.4$ & 0 \\
\hline
\end{tabular}

Abbreviation: $\mathrm{SCl}$, spinal cord injury.

The maximum number of response potentials from one fibre was 12. As shown in Table 2, the number of spontaneously occurring potentials was similar during $10 \mathrm{~s}$ immediately before and immediately after the responses to bladder stimuli (defined by the duration of the multiunit burst in the mean-voltage neurogram).

\section{DISCUSSION}

The major new findings of the present study are that (a) there was a sparse spontaneous activity in individual C fibres in the SCI patients, (b) brief rapid pressure over the urinary bladder could evoke short trains of potentials, but (c) this stimulus-induced single-nerve-fibre activity did not continue after the end of the evoked multiunit burst.

\section{Spontaneous resting activity}

The present results confirm that the integrated neurogram recorded in muscle nerve fascicles below the level of a clinically complete spinal cord lesion contains no spontaneous multiunit bursts. ${ }^{2}$ In all three SCI patients studied, there was, however, a sparse spontaneous activity consisting of individual action potentials.

The potentials recorded in the SCI patients had similar waveforms as those of the vasoconstrictor potentials found in previous 
single-fibre studies in subjects without SCI. ${ }^{67}$ In able-bodied subjects, such potentials occur in the cardiac rhythm, but the lack of cardiac rythmicity in the SCI patients is the expected consequence of their spinal cord lesion. Thus, it is likely that the resting activity of the present study derives from vasoconstrictor fibres. We cannot, however, exclude that occasional action potentials may have originated in single afferent $\mathrm{C}$ fibres. Neither can we exclude that occasional potentials had waveforms and/or properties that were altered by cellular processes secondary to the spinal cord injury: Changes of excitability properties and waveforms after SCI have been reported in human myelinated motor potentials ${ }^{8}$ and in bladder afferent A-delta and C fibre potentials in experimental animals. ${ }^{9}$ In addition, changes of myelinated potential waveforms induced by the electrode causing slowed or blocked propagation have been described, ${ }^{10,11}$ but whether this may also occur for $\mathrm{C}$ fibre potentials is unclear.

Single-fibre potentials evoked by bladder stimuli in SCI patients Rapid pressure over the urinary bladder is known to evoke sympathetic reflex responses in skin and muscle nerves below the level of the lesion in SCI patients but not in intact subjects, ${ }^{1,2}$ indicating an increased excitability of spinal vasoconstrictor neurones in the patients. Only $\sim 60 \%$ of our bladder stimuli evoked single-fibre responses and, when such responses occurred, the number of action potentials varied widely. Presumably, the variability was due at least in part to unintended variations in stimulus intensity, but interindividual differences in excitability cannot be excluded.

The average duration of sympathetic vasoconstrictor multiunit bursts to the muscle vascular bed in able-bodied subjects, with an intact baroreflex regulation, is $\sim 0.6 \mathrm{~s}^{12}$ In comparison, the average duration of multiunit bursts elicited by bladder stimuli in our SCI npatients, lacking baroreflex control of sympathetic outflow, was clearly prolonged (Table 2 ).

Individual nerve fibres fired up to 12 action potentials in response to a bladder stimulation, resembling the 'salvos', that is, bursts of action potentials from a single fibre with high instantaneous firing frequencies, exhibited by muscle vasoconstrictor fibres contributing to prolonged multiunit bursts following premature heart beats in patients with congestive heart failure ${ }^{13}$ or obstructive sleep apnoea. ${ }^{14}$ Theoretically, such high-frequency 'salvos' may transiently generate very high synaptic noradrenaline concentrations, and have also been shown to be associated with high cardiac noradrenaline spillover. ${ }^{15}$ In our SCI patients these 'salvos' occurred exclusively during the multiunit burst, whereas there was no significant difference in the number of spontaneously occurring action potentials during $10 \mathrm{~s}$ immediately before and immediately after the stimulus-induced multiunit burst (Table 2). This finding argues against a prolonged neural response being a major contributing factor to episodes of hypertension after bladder stimuli in SCI patients. Presumably, therefore, stimulus-induced episodes of hypertension in SCI patients are primarily due to an exaggerated vascular responsiveness ${ }^{4,5}$ and/or an increased release of noradrenaline from vasoconstrictor nerve terminals. ${ }^{3}$ Recent studies in experimental animals indicate that after spinal cord lesions both sympathetic nerve traffic and vascular responses to the activity may differ between different vascular beds. ${ }^{16,17}$ Therefore, although evoked sympathetic multiunit responses are similar to skin and muscle nerve activity in human SCI subjects, ${ }^{1,2}$ one cannot automatically assume that the present results from muscle nerves can be extended to all other vascular regions.

\section{Methodological aspects}

In the present study, a fairly large number of action potentials from $\mathrm{C}$ fibres were retrieved from only three SCI patients. This was achieved by quantitative assessment of three measures of action potential waveform: amplitude, duration and slope of the negative downstroke of each potential. Our approach allowed several relatively lowamplitude potentials to be separated from each other in spite of the background noise. The present procedure is in contrast to previous single-unit studies by $u s^{6,7}$ and others ${ }^{18,19}$ in which acceptance of a potential was based mainly on semiquantitative assessment of amplitude and waveform in superimpositions of potentials.

\section{Limitation}

One of our patients received intrathecal infusions of baclofen against spasticity. Baclofen has been shown to affect sympathetic nerve function, ${ }^{20}$ but intrathecal administration yields no systemic concentration of the drug. ${ }^{21}$ Spinal effects of sympathetic nerve function cannot be ruled out, but, as nerve-firing characteristics were similar in all three patients, we do not consider patient medication as a likely confounder in the present study.

To summarize, the present study has shown (a) that there may be a sparse spontaneous activity in individual sympathetic nerve fibres below the level of the lesion in patients with complete cervical spinal cord injury and (b) that stimulation of the urinary bladder evokes only a brief burst of muscle vasoconstrictor impulses in individual nerve fibres. Thus, following the burst there is no continuing (desynchronized) discharge that could contribute to an evoked increase in blood pressure.

\section{DATA ARCHIVING}

There were no data to deposit.

\section{CONFLICT OF INTEREST}

The authors declare no conflict of interest.

1 Wallin BG, Stjernberg L. Sympathetic activity in man after spinal cord injury. Outflow to skin below the lesion. Brain 1984; 107: 183-198.

2 Stjernberg L, Blumberg H, Wallin BG. Sympathetic activity in man after spinal cord injury. Outflow to muscle below the lesion. Brain 1986; 109: 695-715.

3 Karlsson AK, Friberg P, Lönnroth P, Sullivan L, Elam M. Regional sympathetic function in high spinal cord injury during mental stress and autonomic dysreflexia. Brain 1998; 121: 1711-1719.

4 Yeoh M, McLachlan EM, Brook JA. Tail arteries from chronically spinalized rats have potentiated responses to nerve stimulation in vivo. J Physiol 2004; 556: 545-555.

5 Brock JA, Yeo M, McLachlan EM. Enhanced neurally evoked responses and inhibition of norepinephrine reuptake in rat mesenteric arteries after spinal transection. Am J Physiol Heart Circ Physiol 2006; 290: H398-H405.

6 Macefield VG, Vallbo $\AA B$, Wallin BG. The discharge behaviour of single vasoconstrictor motoneurones in human muscle nerves. J Physiol 1994; 481: 799-809.

7 Macefield VG, Elam M, Wallin BG. Firing properties of single postganglionic sympathetic neurones. Auton Neurosci 2002; 95: 146-159.

8 Lin CS-Y, Macefield VG, Elam M, Wallin BG, Engel S, Kiernan M. Axonal changes in spinal cord injured patients distal to the site of injury. Brain 2007; 130: 985-994.

9 DeGroat WC, Yoshimura N. Changes in afferent activity after spinal cord injury. Neurourol Urodyn 2010; 29: 63-76.

10 Vallbo $\AA$ B. Prediction of propagation block on the basis of impulse shape in single unit recordings from human nerves. Acta Physiol Scand 1976; 97: 66-74.

11 Torebjörk HE, Hallin RG, Hongell A, Hagbarth K-E. Single unit potentials with complex waveform seen in microelectrode recordings from the human median nerve. Brain Res 1970; 24: 443-450.

12 Wallin BG, Burke D, Gandevia S. Coupling between variations in strength and baroreflex latency of sympathetic discharges in human muscle nerves. J Physiol 1994; 474: 331-338.

13 Elam M, Macefield V. Multiple firing of single muscle vasoconstrictor neurons during cardiac dysrhythmias in human heart failure. J Appl Physiol 2001; 91: 717-724. 
14 Elam M, McKenzie D, Macefield V. Mechanisms of sympathoexcitation: single-unit analysis of muscle vasoconstrictor neurons in awake OSAS subjects. J App/ Physiol 2002; 93: 297-303.

15 Lambert EA, Schlaich MP, Dawood T, Sari C, Chopra R, Barton DA et al. Single-unit muscle sympathetic nervous activity and its relation to cardiac noradrenaline spillover. J. Physiol 2011; 589: 2597-2605.

16 McLachlan EM, Brock JA. Adaptations of peripheral vasoconstrictor pathways after spinal cord injury. In: Weaver LC and Polosa C (eds) Progress in Brain Research, Vol 152. Elsevier, 2006, pp 289-297.

17 McLachlan EM. Diversity of sympathetic vasoconstrictor pathways and their plasticity after spinal cord injury. Clin Auton Res 2007; 17: 6-12.
18 Lambert EA, Dawood T, Barton DA, Esler MD, Lambert G. Single-unit analysis of sympathetic nervous discharges in patients with panic disorder. J. Physiol 2006; 570 637-643.

19 Murai H, Takata S, Maruyama M. The activity of a single muscle sympathetic vasoconstrictor nerve unit is affected by physiological stress in humans. Am J Physio Heart Circ Physiol 2006; 290: H853-H860.

20 Bowery NG, Doble A, Hill DR, Hudson AL, Shaw JS, Turnbull MJ et al. Bicucullineinsensitive GABA receptors on peripheral autonomic nerve terminals. Eur J Pharmacol 1981; 71: 53-70.

21 Brennan PM, Whittle IR. Intrathecal baclofen therapy for neurological disorders: a sound knowledge base but many chanllenes remain. Brit J Neurosurg 2008; 22: 508-519. 